\title{
Evaluación de una prueba de ensayo inmunoabsorbente ligado a enzimas de competencia (ELISA-C) para el diagnóstico serológico de la brucelosis bovina.
}

\section{Artículo Original}

Alfredo Dájer-Abimerhi ${ }^{1}$, Edwin J. Gutiérrez-Ruiz ${ }^{1}$, Delfina de las M. Zapata-Villalobos ${ }^{1}$, Eduardo M. SierraLira $^{1}$, Elsie I. Cámara-Gamboa ${ }^{1}$.

${ }^{1}$ Unidad de Servicios Auxiliares para el Diagnóstico, Facultad de Medicina Veterinaria y Zootecnia, Universidad Autónoma de Yucatán, Mérida, Yucatán, México.

\section{RESUMEN.}

Introducción. La brucelosis es una zoonosis a nivel mundial que además causa severas pérdidas a la ganadería por lo que es necesario tener pruebas de laboratorio específicas, sensibles y sencillas para establecer medidas de prevención y control efectivas. Material y Métodos. Se probaron 724 sueros de bovino del banco del Depto. de Inmunología de la FMVZ-UADY, con un kit experimental de ELISAC. El punto de corte se determinó mediante un análisis ROC y una gráfica de dispersión. La concordancia entre la ELISA-C y Fijación de complemento (FC) se determinó con la prueba de Kappa. La sensibilidad y la especificidad se determinaron usando un cuadro de contingencia.

Resultados. El punto de corte (PC) encontrado fue de $47 \%$ de inhibición. La concordancia de la CELISA con relación a la prueba de FC fue de 0.80 para el total de sueros, 0.84 para sueros de animales no vacunados y de 0.72 para los vacunados. La sensibilidad fue de $92.27 \%$ (95\% IC. 88.51-96.03) para el total de los sueros, 93.86\% (95\% IC. 89.4598.27) para sueros de animales no vacunados y $90 \%$ (95\% IC. 83.43-96.57) para los vacunados. La especificidad fue de $91.89 \%$ (95\% IC. 89.56-94.21) para el total de los sueros, $94.26 \%$ (95\% IC. 92.0396.49) para sueros de animales no vacunados y 83.04\% (95\% IC. 76.08-89.99) para los vacunados. Discusión. El punto de corte encontrado difiere del obtenido $(30 \%)$ por el fabricante del Kit. La concordancia de la ELISA-C y FC fue alta pero su especificidad y sensibilidad con el PC de $>47 \%$ de inhibición, no la hacen una mejor prueba que FC; sin embargo, moviendo este PC se puede mejorar la especificidad o sensibilidad dependiendo de los objetivos de la campaña para el control/erradicación de la brucelosis bovina.

(Rev Biomed 2003; 14:23-28)

Palabras clave: ELISA de competencia, fijación de complemento, brucelosis bovina.

Solicitud de sobretiros: M. en C. Edwin J. Gutiérrez-Ruiz, Depto. de Virología, Fac. de Medicina Veterinaria y Zootecnia, Universidad Autónoma de Yucatán, Mérida, Yucatán, México. Tel.: (999) 942-32-00 E-mail: gruiz@tunku.uady.mx 


\section{A Dájer-Abimerhi, EJ Gutiérrez-Ruiz, D de las M Zapata-Villalobos y col.}

\section{SUMMARY.}

Evaluation of a competitive enzime linked immunosorbant assay for the serological diagnosis of bovine brucellosis.

Introduction. Brucellosis is a worldwide zoonotic disease, that causes losses to cattle production, so its necessary to have simple, sensitive and specific diagnostic tests to establish effective prevention and control measures.

Material and Methods. 724 bovine sera were tested with an experimental competitive enzime linked immunoabsorbant assay (C-ELISA); the cut-off point was determined using a ROC analysis and a scattergram. Agreement between the C-ELISA and the complement fixation test (CFT) was determined with the Kappa test. Sensitivity and specificity were also determined.

Results. The optimum cut off-point was $47 \%$ inhibition.Agreement between C-ELISA and CFT was 0.8 for all sera, 0.84 for sera from non vaccinated animals and 0.72 for sera from vaccinated animals. Sensitivities were $92.27 \%$ (confidence intervals $95 \%$ (CI) 88.51-96.03) for all sera; 93.86\% (CI 95\% 84.45-98.27) for sera from non vaccinated animals and 90\% (CI 95\% 83.43-96.57) for sera from vaccinated animals. Specificities were $91.89 \%$ (CI 95\% 89.56-94.21) for all sera; $94.26 \%$ (CI 95\% 92.05-96.49) for sera from non vaccinated animals and $83.04 \%$ (CI 95\% 76.08-89.99) for sera from vaccinated animals.

Discussion. The optimum cut-off determined was different from that recommended by the kit producer. Agreement between C-ELISA and CFT was high but the sensitivities and specificities with the $47 \%$ inhibition cut-off point do not make the C-ELISA a better test than CFT. However, moving the cut-off point can improve sensitivity or specificity depending on the objectives of the control/erradication compaing for bovine brucellosis. (Rev Biomed 2003; 14:23-28)

Key words: Competitive ELISA, complement fixation test, bovine brucellosis.

\section{INTRODUCCIÓN.}

La brucelosis es una enfermedad que se mantiene como una de las zoonosis de mayor distribución en el mundo, aunque en pocos países se ha logrado erradicar y en muchos se encuentre bajo control. El impacto económico que tiene en la empresa pecuaria es alto, debido a la reducción en la fertilidad del hato, gran número de abortos, nacimiento de becerros débiles, bajo peso al destete y reducción en la producción de leche (1,2-5).

La Norma Oficial Mexicana para la campaña nacional contra la brucelosis en los animales (NOM) vigente, establece tres pruebas serológicas oficiales: Rosa de Bengala (RB), Rivanol y Fijación de complemento (FC) $(4,5)$.

Estas pruebas poseen características de elaboración y/o de sensibilidad y especificidad que hacen que se sigan buscando otras alternativas para el diagnóstico de la brucelosis bovina. La prueba de RB aunque tiene una alta sensibilidad relativa (100\%) al compararla con FC, su baja especificidad la hace solamente útil como prueba tamiz $(4,5)$. En comparación, la prueba de Rivanol tiene una especifidad relativa alta (100\%) comparada con FC, pero una sensibilidad relativa de $86 \%$, lo que la hace útil como prueba complementaria $(6,7)$. La prueba de FC es considerada de gran valor diagnóstico por su alta sensibilidad y especificidad (cercanas al 99\%); sin embargo, es de uso limitado debido al tiempo que se invierte en su estandarización, así como su complejidad técnica, que requiere de personal calificado $(6,8,9)$.

En la actualidad es necesario la implementación de pruebas serológicas sencillas y confiables que nos ayuden a discriminar los animales infectados con $B$. abortus de aquellos infectados con bacterias que reaccionan en forma cruzada y los vacunados con la cepa 19 de B. abortus, lo cual se ha logrado con diferentes sensibilidades y especificidades dependiendo del antígeno y anticuerpos monoclonales utilizados en diferentes ELISA-C (10-12), ya que a pesar que esta cepa esta descontinuada en México para vacunar a los bovinos, todavía quedan en el hato bovino estatal y nacional animales que fueron

\section{Revista Biomédica}




\section{Evaluación de una ELISA-C para el diagnóstico de brucelosis bovina.}

vacunados. La obtención de una prueba de estas características permitiría la implementación de medidas de control y erradicación más eficientes para esta enfermedad. El objetivo de este estudio es evaluar una prueba, que potencialmente puede diferenciar animales vacunados de los infectados con cepas de campo, ELISA-C bajo condiciones del estado de Yucatán, México.

\section{MATERIALES Y MÉTODOS. Sueros.}

Se usaron 724 sueros de bovinos adultos (mayores de 2 años de edad), de ambos sexos, del banco del departamento de Inmunología de la Facultad de Medicina Veterinaria y ZootecniaUniversidad Autónoma de Yucatán (FMVZ-UADY) que se recabo de septiembre de 1997 a marzo de 1998 con las siguientes características:

1) Sueros de animales no infectados con $B$. abortus.

418 sueros provenientes de hatos libres de brucelosis y donde no se aplicaba vacuna contra la enfermedad.

112 sueros provenientes de hatos libres de brucelosis y donde se aplicaba vacuna de la cepa 19 contra la enfermedad.

2) Sueros de animales infectados con $B$. abortus.

114 sueros provenientes de hatos infectados con brucelosis (con por lo menos $2 \%$ de reactores a la prueba de FC y con la presencia de signos como abortos), donde no se aplicaba vacuna contra la enfermedad y resultados positivos a la pruebas de $\mathrm{RB}$ y FC.

80 sueros provenientes de hatos infectados con brucelosis (con por lo menos $2 \%$ de reactores a la prueba de fijación de complemento y con la presencia de signos como abortos), donde se aplicaba vacuna de la cepa 19 contra la enfermedad y que resultaron positivos a la prueba de RB y FC por lo menos 1 año después de aplicada la vacuna.

\section{Pruebas serológicas.}

La prueba de FC se realizó de acuerdo al método descrito por el Laboratorio Central Veterinario de Weybridge, Inglaterra (CVLWeybridge), con la única excepción de que las muestras se corrieron en diluciones dobles de 1:2 hasta 1:256 debido al fuerte efecto de prozona encontrado previamente con bajas diluciones (9). Las concentraciones de unidades internacionales se derivaron de cuadros estándares. El antígeno y los sueros control positivo y negativo se obtuvieron del CVL-Weybridge; el complemento y la hemolisina se obtuvieron de Tissue Culture Services, Bolton, Inglaterra. La prueba de ELISA-C se realizó con un kit diagnóstico experimental, que utiliza como antígeno el lipopolisacarido liso de Br. abortus, un anticuerpo monoclonal (M84), un suero control positivo fuerte (inhibe la capacidad de unión del M84 entre 95 y 99\%), un suero control positivo débil de un animal vacunado (positivo en ELISA indirecto pero que solo inhibe el M84 entre 5 y 20\%), un suero control negativo que no compite con el M84 (0-5\% de inhibición) y un control de conjugado (solo diluyente), el conjugado consistente en IgG anti-ratón de cabra con peroxidasa de rabano picante y una solución de sustrato/ cromógeno (peroxido de hidrógeno/2,2' - azinobis (ácido tiazolino sulfónico-3 etilobenceno) [ABT] siguiendo el protocolo de los fabricantes $(11,12)$.

\section{Análisis.}

El análisis de los resultados se llevó a cabo en el programa Epi Info Versión 6.1 (13), el punto de corte de laELISA-C se determinó mediante un análisis ROC y una gráfica de dispersión, utilizando los programas de computo Excel Versión 6.0 (14) para windows 97 y Med Calc Versión 3.0 (15).

En la evaluación de la prueba de ELISA-C se determinó su concordancia con la prueba de FC, utilizando la prueba de Kappa, la cual indica el grado de concordancia entre las pruebas, esto es, un valor de Kappa 0 indica no relación entre las pruebas, siendo el valor 1 indicador de una correlación perfecta (16).

La sensibilidad y especificidad de la ELISA-C con sus intervalos de confianza, se determinaron utilizando los sueros de las poblaciones descritas anteriormente como el patrón de comparación (16). 


\section{A Dájer-Abimerhi, EJ Gutiérrez-Ruiz, D de las M Zapata-Villalobos y col.}

Tanto la concordancia como la sensibilidad y especificidad de la ELISA-C y FC se determinaron para los grupos de sueros provenientes de animales vanunados, no vacunados y con el total, utilizando el programa de computo Episcope Versión 1.0 (17).

\section{RESULTADOS.}

El punto de corte para la ELISA-C se determinó en $>46 \%$ de Inhibición, donde cualquier suero con un valor $\geq 47 \%$ se consideró como positivo. El análisis ROC y la dispersión de resultados de donde se obtuvo el punto de corte se pueden observar en las figuras 1 y 2.

La concordancia de la ELISA-C con relación a la prueba de FC fue de 0.8 para el total de los sueros, 0.84 para los sueros de animales no vacunados y 0.72 para los de animales vacunados.

La sensibilidad de la ELISA-C fue de $92.27 \%$ (95\% IC 88.51-96.03) para el total de los sueros; 93.86\% (95\% IC 89.45-98.27) para sueros de animales no vacunados y $90 \%$ (95\% IC 83.43 96.57) para sueros de animales vacunados.

La especificidad de la ELISA-C fue de 91.89\% (95\% IC 89.56-94.21) para el total de los

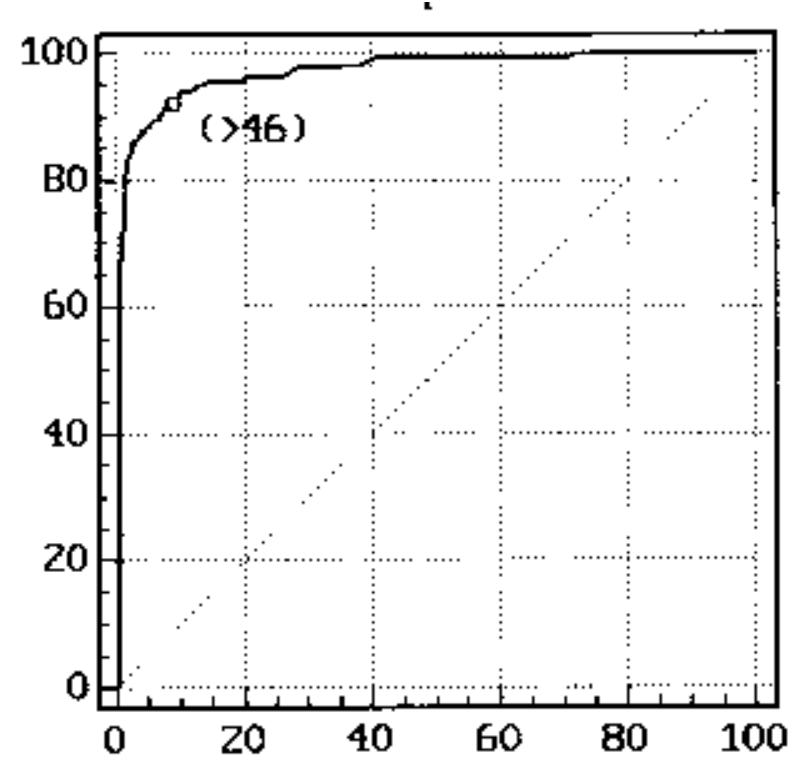

Figura 1.- Curva de análisis ROC para determinar el punto de corte con la mejor sensibilidad y especificidad, utilizando sueros de bovino ( $\mathrm{n}=724)$ del estado de Yucatán, México, para la prueba de ensayo inmunoabsorbente ligado a enzimas de competencia (ELISA-C), elaborado por el Animal Diseases Research Institute de Canadá.

Revista Biomédica de animales no vacunados y $83.04 \%$ (95\% IC 76.0889.99) para sueros de animales vacunados.

\section{DISCUSIÓN.}

El punto de corte encontrado en el presente estudio ( $\geq 47 \%$ de Inhibición) es más elevado que el recomendado por el fabricante del kit que es del $30 \%$ de Inhibición, esto probablemente puede explicarse por el hecho de que en un clima tropical como en el estado de Yucatán, la presencia de microorganismos que pueden tener una reacción cruzada de anticuerpos con B. abortus como especies de Salmonella y Yersinia (18-19) es mayor a la que se podría encontrar en climas templados como el de Canadá en donde se origina esta prueba de ELISA-C.

Otra posible razon de la elevación del punto de corte es la inclusión de sueros de animales vacunados provenientes de hatos libres. Esto mismo se refleja en

POSITIVO NEGATIVO

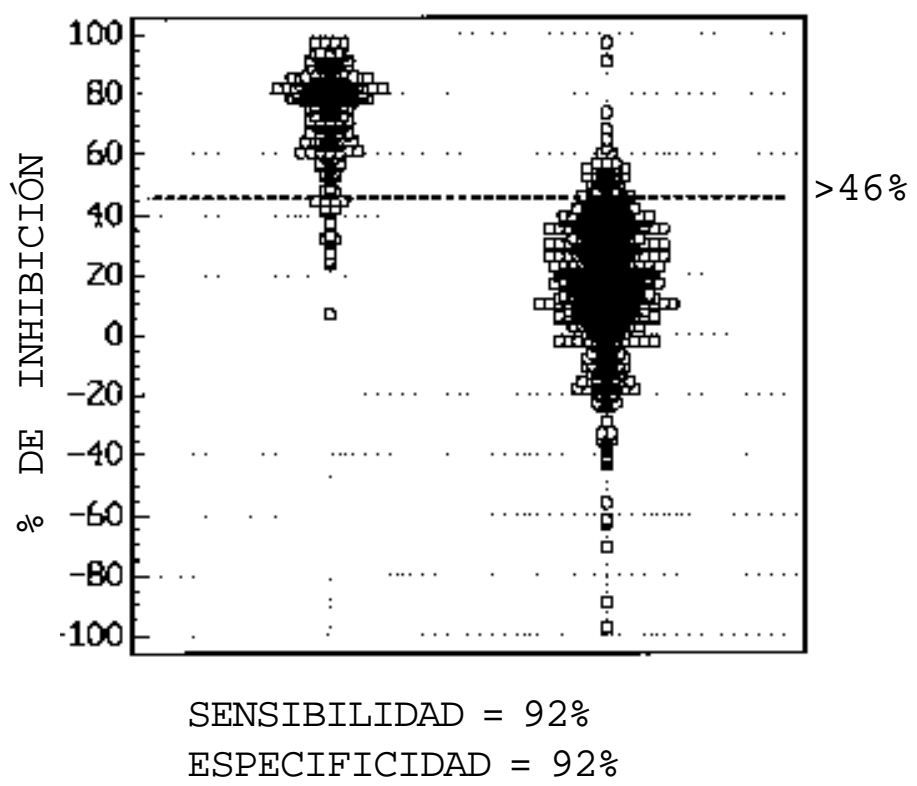

Figura 2.- Dispersión de los sueros positivos y negativos a anticuerpos contra Brucella abortus de acuerdo a su porcentaje de inhibición en la prueba de ensayo inmunoabsorbente ligado a enzimas de competencia (ELISA-C), elaborado por el Animal Diseases Research Institute de Canadá, para la determinación del punto de corte de la misma con sueros de bovino del estado de Yucatán, México. 


\section{Evaluación de una ELISA-C para el diagnóstico de brucelosis bovina.}

la concordancia entre la ELISA-C y FC donde a pesar que siempre se encontró una alta concordancia $(0.73,0.80$ y 0.83$)$, la diferencia entre los sueros obtenidos de animales vacunados $(0.73)$ y de animales no vacunados $(0.83)$ es considerable.

La sensibilidad de ELISA-C comparada con FC fue más alta para los sueros de los animales no vacunados con respecto de los animales vacunados aunque para los dos grupos fue relativamente baja, lo cual hace que la ELISA-C con el punto de corte seleccionado no sea recomendable para las condiciones del estado pues no identifica algunos animales infectados los cuales pueden mantener la infección en el hato.

La especificidad de la ELISA-C comparada con FC fue más alta para sueros provenientes de animales no vacunados en relación con los vacunados lo que puede ser indicativo de que los anticuerpos vacunales por cepa 19 de algún modo si pueden ser detectados por la ELISA-C en contraste con lo reportado previamente por los fabricantes; sin embargo, la capacidad de la ELISA-C para detectar a los verdaderos positivos y negativos se incrementará conforme sean retirados los animales vacunados de la población (11).

Es importante señalar que a pesar de que con el punto de corte seleccionado se obtiene la máxima sensibilidad y especificidad combinadas lo cual es útil para la evaluación, moviendo el punto de corte de esta prueba se puede incrementar la sensibilidad o la especificidad, lo que permitiría usar esta prueba en diferentes etapas de la campaña de control y erradicación de la brucelosis bovina en nuestro estado. Desde luego es necesario volver a evaluar la prueba en un futuro cuando ya no haya animales vacunados con la cepa 19 de B. abortus pero si con la cepa RB51, en el hato bovino estatal.

\section{REFERENCIAS.}

1.- Alvarez E. Situación de la brucelosis en América: Panorama general. En: SAGAR-UNAM editores. Memorias del III Foro Nacional de Brucelosis; 1998. pp 23-31.

2.- Corbel MG. Brucelosis. En: Laing JA, Brinkley WR,
Wagner WC, editores. Fertilidad e Infertilidad en la práctica veterinararia. 4a ed. México: Interamericana-McGraw-Hill; 1996. pp. 201-36.

3. - Bernués A, Manrique E, Maza M. Economic evaluation of bovine brucellosis and tuberculosis erradication programmes in a mountain area of Spain. Prev Vet Med 1997; 30:137-49.

4.- López MA, López SR, Ocampo DA, González DI. Brucelosis avances y perspectivas. México: INDRESubsecretaría de Coordinación y Desarrollo- SSA; 1991.

5.- Diario Oficial . Normas de la Campaña Nacional contra la Tuberculosis y Brucelosis en México. México; 1996, pp. 4566.

6.- Central Veterinary Laboratory (CVL). Standard Laboratory Techniques for Brucellosis Diagnosis. Inglaterra (Weybridge): Ministry of Agriculture, Fisheries and Food; 1991.

7.- Mejía TC, Luna ME. Descripción y generalidades de la brucelosis. En: Secretaria de Agricultura y Ganaderia RuralEscuela de Médicos Veterinarios Zootecnistas editores. Manual de actualización técnica para la aprobación del médico veterinario como unidades de verificación en tuberculosis bovina y brucelosis. México; 1996. p. 43-46.

8.- Dájer AA, Gutiérrez RE, Zapata VD, Villegas PS. Uso de las pruebas de ensayo inmunoabsorbente ligado a enzimas y aglutinación con Rivanol para el diagnóstico de Brucelosis Bovina en Yucatán. Vet Méx 1998; 29: 167-71.

9.- Dájer-Abimerhi A, Gutiérrez-Ruiz E, Zapata-Villalobos D, Honhold N, Villegas-Pérez S. Comparación de cinco pruebas serológicas para la detección de anticuerpos contra Brucella abortus y reporte preliminar del porcentaje de reactores positivos en hatos bovinos en Yucatán, México. Rev Biomed 1995; 6:84-90.

10.-Wright PF, Nielsen KH, Kelly WA. Primary binding techniques for the serodiagnosis of bovine brucellosis: Enzyme immunoassay. En Nielsen KH, Duncan JR, Editores. Animal brucellosis. Boca Ratón: CRC Press; 1990. p. 199235 .

11.- Nielsen K, Gall D, Kelly W, García M. Enzyme immunoassay, application to diagnosis of bovine brucellosis. Canadá; Animal Diseases Research Institute, Agriculture and Agri-Food Canada: 1992. pp 35-43.

12.- Nielsen KH, Kelly L, Gall D, Nicoletti P, Kelly W. Improved 


\section{A Dájer-Abimerhi, EJ Gutiérrez-Ruiz, D de las M Zapata-Villalobos y col.}

competitive immunoassay for the diagnosis of bovine brucellosis, Vet Immunol Immunopath 1995; 45:285-91.

13.- Center Disease Control. Epi Info Versión 6.1. Organización Mundial de la Salud. E.U.

14.- Microsoft Corporation. Excel Versión 6.0. E.U.

15.- Animal Diseases Research. Med Calc Versión 3.0. Belgium.

16.- Martín W, Meek A, Willenberg P. Veterinary epidemiology: Principles and methods. Iowa: Iowa State Press; 1987. p. 343.

17.- Agger JF, Noordhuizen J, Willeberg P, Van Voorthuysen PF. Episcope Versión 1.0. Agricultural University. Netherlands.

18.- Blood DC, Henderson JA, Radostitis OM. Medicina Veterinaria. 6a ed. México: Interamericana; 1986. pp 662-673.

19.- Food and Agricultural Organization (FAO)/World Health Organization (WHO)/Organization International of Epizoties (OIE). Report 1986: Joint FAO/WHO Expert Committee on Brucellosis, EUA : WHO Technical Report Series; 1986.

\section{Revista Biomédica}

